\title{
ARTICLE
}

\section{In-situ radioactivity measurement for the site release after decommissioning of nuclear power plants}

\author{
Tadao Tanaka*, Taro Shimada and Takenori Sukegawa \\ Japan Atomic Energy Agency, 2-4 Shirakata, Tokai-mura, Naka-gun, Ibaraki-ken, 319-1195, Japan
}

\begin{abstract}
According to a basic policy of Japan, nuclear power plant sites are allowed to be released from nuclear safety regulations after the plant are decommissioned. It is necessary to confirm that there is no significant radioactivity remaining on the sites, before the site release. In the present study, we proposed a conservative and reasonable evaluation method of radioactivity remaining on sites of decommissioned nuclear power plant, and confirmed validity of the evaluation method by an in-situ measurement using a germanium semiconductor detector (Ge detector). Cobalt 60 (Co-60) was taken up as one of the typical radionuclides for nuclear power plant. In the evaluation concept, all of Co-60, which is distributed across the area of interest (AOI), were assumed to be the single point source located at the furthest position on the surface of AOI from the Ge detector. In such a configuration, the shortest period needed for detection (SPD) of Co-60 was predicted by the calculation with EGS5 code. The minimum detectable time is most conservative detection limit for the all of Co-60. If the actual measurement time to detect Co-60 distributed across AOI became greater than SPD, the fact supports that there is no significant Co-60 on AOI.
\end{abstract}

Keywords: site release; decommissioning; in-situ measurement; Cobalt 60; portable germanium semiconductor detector

\section{Introduction}

According to a basic policy of Japan, nuclear power plant (NPP) site is allowed to be released from nuclear safety regulations after the plants are decommissioned. It is necessary to confirm that there is no significant radioactivity remaining on the sites, for the site release beforehand [1]. In the present study, we propose a conservative and reasonable evaluation method of radioactivity remaining on sites of decommissioned NPP, and confirmed validity of the evaluation method by an examination.

Cobalt 60 (Co-60) and cesium 137 (Cs-137) are the typical radionuclides for NPP. NPPs through Japan are widely affected by fallout radioactive cesium from severe accident of NPP in Fukushima. Thus Cs-137 is not available for key nuclide to be discharged from NPP. After the severe accident, Co-60 will be most effective key nuclide [2].

The in-situ metrology with portable germanium semiconductor detector (Ge detector) has been applied to measure radioactivity remaining on the sites [3]. The Ge detector has no necessity of the preprocessing and the waiting for measurements, can evaluate in real time on the sites, and can identify the nuclide by emission energy. Moreover, it is a flexible metrology having a lot of advantages, for example to measure the large area and to secure the representativeness $[1,3,4]$.

\section{Concept of the evaluation method}

\subsection{Check process for the site release}

Referential knowledge on radioactivity measurement and evaluation process for the site release after decommissioning NPP was proposed in MARSSIM published from USNRC [1]. Decommissioning entrepreneur must perform final status survey on the area of interest (AOI) after decontamination under decommissioning license plan, in order to prove that there is no significant radioactivity remaining on the sites. Regulatory agency must judge the results presented from decommissioning entrepreneur, from view point of validity of planning, equipment, measurement method, results, analytical method, and so on. Further regulatory agency has to independently check by inspection survey, even if the results presented from decommissioning entrepreneur are acceptable.

On the basis of above process, purpose of the inspection survey by regulatory agency is to reconfirm that there is no significant radioactivity remaining on the sites.

*Corresponding author. Email: tanaka.tadao26@jaea.go.jp 


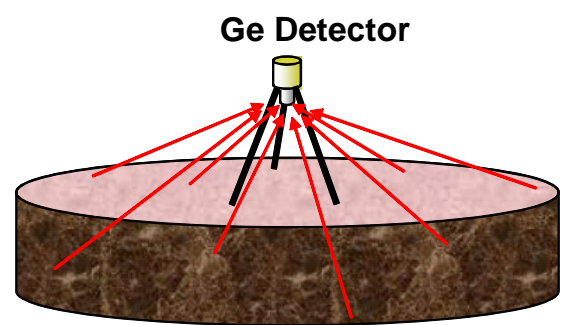

All of the Co-60 are in reality distributed across the area of interest
Application of conservative radioactivity conversion coefficient

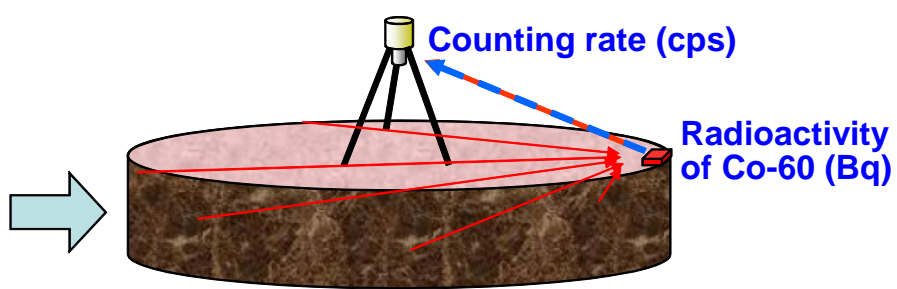

All of the Co-60 are assumed to be presented as a single point source located at the furthest

Figure 1. Concept of inspection survey for the conservative judgment.

\subsection{Premise condition on the inspection survey}

As the premise condition on the inspection survey,

- Final status survey on the sites had been carried out by decommissioning entrepreneur, and

- Regulatory agency had judged that there is no significant Co-60 on the sites, by using the results presented from decommissioning entrepreneur.

\subsection{Evaluation method for the inspection survey}

The concept of proposed inspection survey is drawn in Figure 1. It is assumed that, Co-60 pollution is attributable to ground surface deposition. All of the Co-60, which is in reality distributed across AOI, are assumed to be presented as a single point source located at the furthest position from a Ge detector on the ground surface of AOI. In such a configuration, the shortest counting period needed for detection (SPD) of the Co-60 point source is predicted by a calculation, as described in the section bellow. If the radiation from $\mathrm{Co}-60$ in $\mathrm{AOI}$ is not detected within period longer than SPD, it proves that the radioactivity of Co-60 remaining in AOI is lower than that of Co- 60 point source most difficult to detect. The difference from previous method is that it is not necessary to measure exactly the absolute radioactivity.

In actual situation, the Ge detector also counts the radiation from outside of AOI, as shown in Figure 2.
The influences of radiation from outside apparently increase with decreasing radius of AOI [3]. Thus, the evaluation concept by the inspection survey is to provide more conservative judgment in actual situation.

\section{Prediction of SPD for Co-60 point source set up at the furthest position}

\subsection{Prediction method}

In general radiation measurement, relationship between detection limit counting rate $N_{\mathrm{L}}$ (cps) and counting time $t(\mathrm{sec})$ can be approximated to Eq. (1).

$$
t=a \times N_{L}^{-b}
$$

where $a$ and $b$ represent constants of correlation.

The $N_{\mathrm{L}}$ is dominated by efficiency of detector and intensity of radiation source. Substituting the detection limit counting rate with detection limit radioactivity $A_{\mathrm{L}}$ $(\mathrm{Bq})$ and radioactivity conversion coefficient $K(\mathrm{~Bq} / \mathrm{cps})$, we obtain

$$
t=a \times\left(A_{L} / K\right)^{-b}
$$

The $K$ corresponding to configuration in Figure 1 is calculated from the particle transport simulation using the Electron-Gamma Shower code, version 5 (EGS5) [5]. PSD can be theoretically predicted by using $A_{\mathrm{L}}$ and $K$ in each testing condition.

\section{Ge Detector}
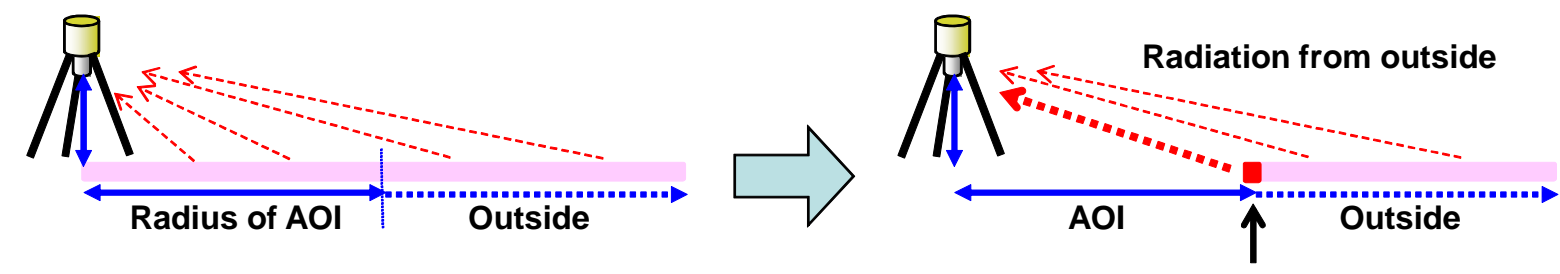

All of the Co-60 in AOI

Figure 2. Influence of radiation form outside of area of interest (AOI). 


\subsection{Example for prediction of SPD}

The German regulation criteria of Co-60 radioactivity for site release are $0.03 \mathrm{~Bq} / \mathrm{g}$ [6]. On the other hand, the clearance level of Co-60 under Japanese regulation criteria is $0.1 \mathrm{~Bq} / \mathrm{g}$ [7]. These regulation criteria values may be reference of regulation criteria of Co- 60 for the site release. If Co-60 of $0.1 \mathrm{~Bq} / \mathrm{g}$ is uniformly distributed into the depth of $0.54 \mathrm{~cm}$ from ground surface in a soil layer of $1.6 \mathrm{~g} / \mathrm{cm}^{3}$ in density, there is $2.7 \times 10^{5} \mathrm{~Bq}$ of Co-60 on AOI of $10 \mathrm{~m}$ in radius. This value corresponds to $A_{\mathrm{L}}$ in Eq. (2). The $K$ corresponding to $1,173 \mathrm{keV}$ $(\gamma$-ray emission energy of Co-60) for a Ge crystal $(60$ $\mathrm{mm}$ in diameter, $45.5 \mathrm{~mm}$ in height, $30 \%$ in efficiency) is calculated to $3.3 \times 10^{6} \mathrm{~Bq} / \mathrm{cps}$ by using the EGS5 code.

Average values of $a$ and $b$ in Eq. (2) for the Ge detector have been obtained by fitting Eq. (1) into time-dependent changes of the detection limit counting rate acquired from background measurements, to be $a=$ 2.51 and $b=1.93$ [8]. In the configuration mentioned above, SPD is decided to be $313 \mathrm{sec}$, using Eq. (2) and the obtained $a, b, A_{\mathrm{L}}$ and $K$ values. If radiation from Co-60 is not detected by actual measurement within 313 sec, it can be conservatively and rationally reconfirmed that there is no significant Co-60 in AOI of $10 \mathrm{~m}$ in radius.

\section{In-situ inspection survey test}

\subsection{Method of inspection survey test}

Based on the configuration mentioned above, an inspection survey test using radiation source was performed in-situ. No Co-60 was detected by background measurement for $12,000 \mathrm{sec}$ in the test site. Radiation source of 2.7 x 10 $10^{5} \mathrm{~Bq}$ was prepared as Co-60 point source. The point source was sealed in a column type polyethylene container of $5 \mathrm{~cm}$ in diameter and 5 $\mathrm{cm}$ in height. The point source was placed at a fixed distance of $10 \mathrm{~m}$ from the Ge detector (Refer to Figure 3). The Ge detector was set up at $1 \mathrm{~m}$ in height above the ground level. The time-dependent changes of the detection limit counting rate and net counting rate at the peak of $1,173 \mathrm{keV}$ were analyzed.

\subsection{Results on the inspection survey test}

Time-dependent changes of the detection limit counting rate and the peak counting rate are shown in Figure 4. The measurements were carried out five runs under the same testing condition. When the peak counting rate was greater than the detection limit counting rate, it is regarded that the Co-60 point source was detected. The actual detection time was nearly 300 sec in Figure 4. The actual detection time in each test run was decided by according to Figure 5, where the peak counting rate exceeded firstly the detection limit counting rate. Average value of the actual detection time through five runs was $326 \mathrm{sec}$. The predicted SPD (313 sec) was nearly equal to the average value.

According to the evaluation concept, the time A must decrease when the point source is not kept at the furthest position. The point source was moved to distance of $5 \mathrm{~m}$ and $7 \mathrm{~m}$ from the Ge detector, and was measured. Average value of the time A was also decided to $21 \mathrm{sec}$ in the case of $5 \mathrm{~m}$ and $78 \mathrm{sec}$ in the case of $7 \mathrm{~m}$ respectively. It was confirmed, by the in-situ test, that the detection time became short clearly in the case that Co-60 was not kept at the furthest position.

The results suppose that the prediction procedure of SPD for radiation source set up at the furthest position was reliable and reasonable.

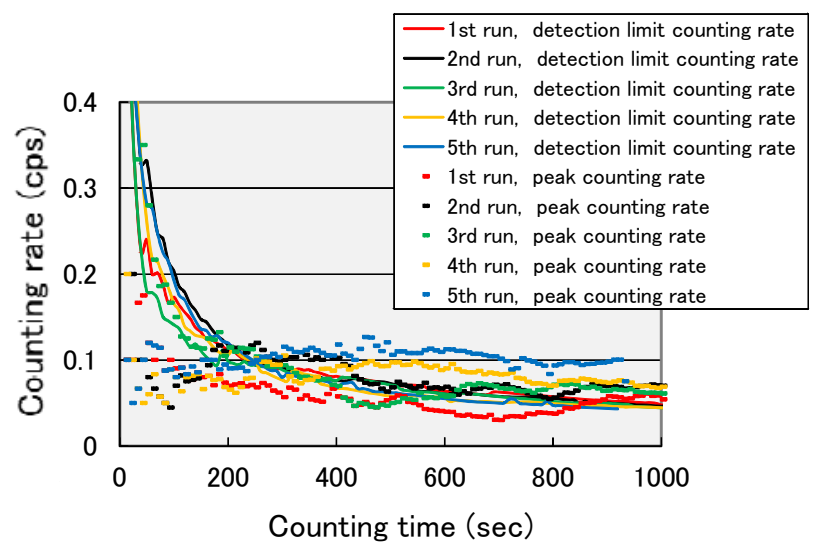

Figure 4. Time-dependent changes of detection limit counting rate and peak counting rate in inspection survey
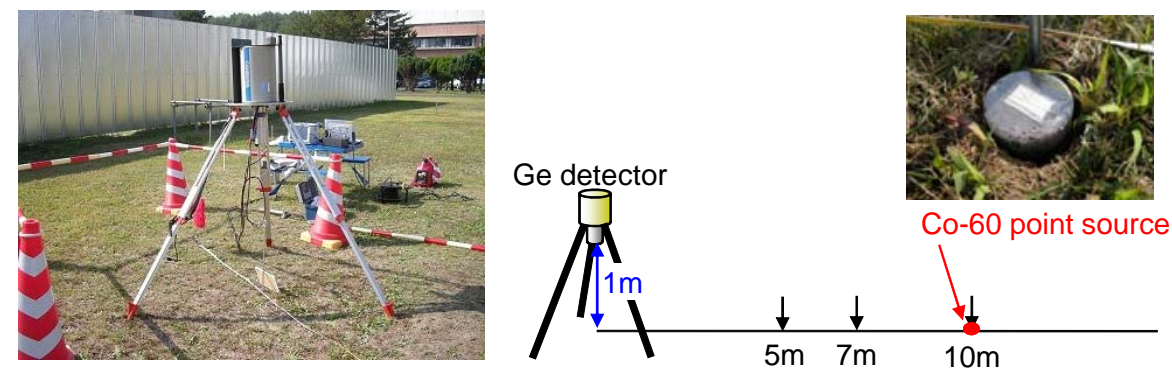

Figure 3. Schematic view of inspection survey test. 

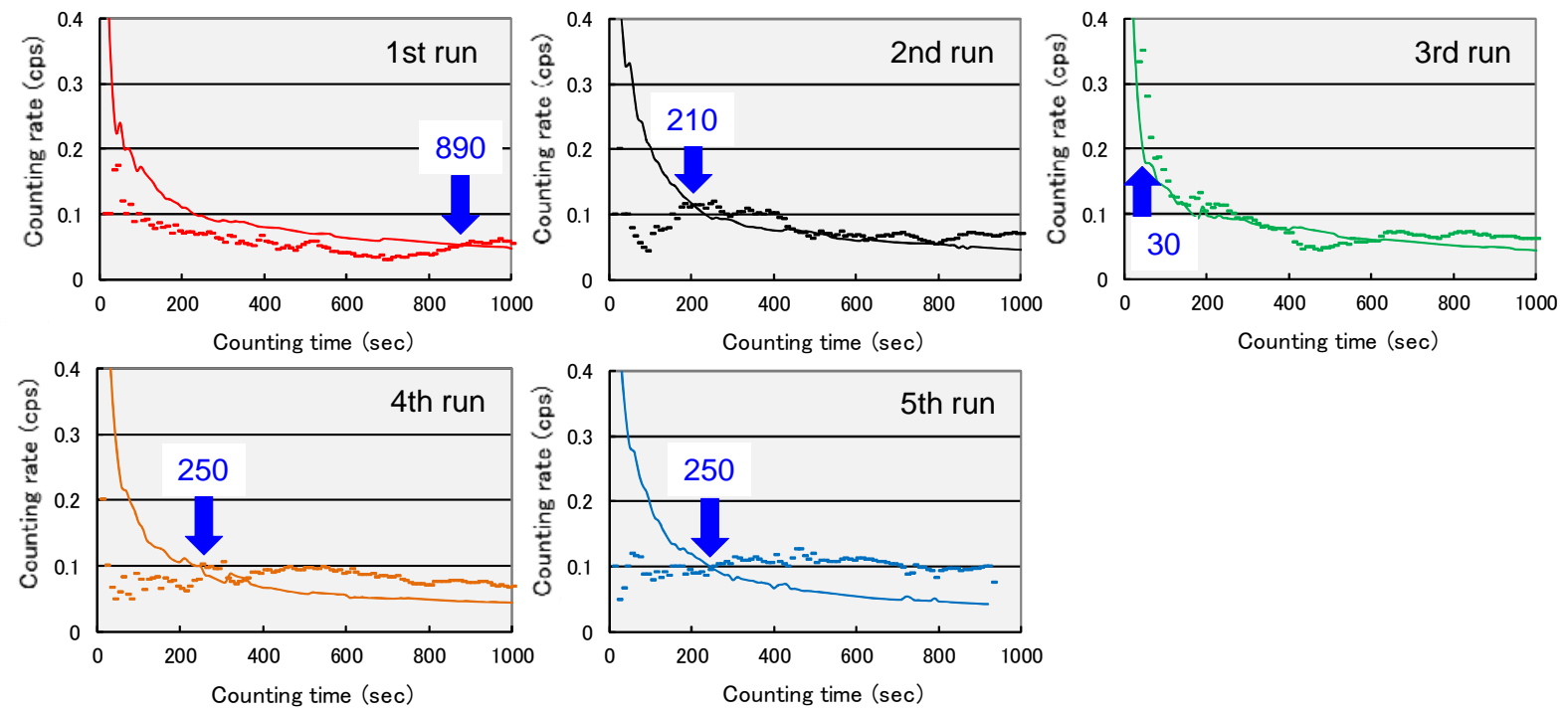

Figure 5. Decision of actual counting time.

\section{The evaluation procedure for the inspection survey}

A standardized procedure for the inspection survey is described as follows.

1) Set up AOI.

2) Set up the referential Co-60 criteria for AOI.

3) Estimation of the total radioactivity of Co-60 on AOI.

4) Set up layout of $\mathrm{Ge}$ detector in AOI.

5) Decision of constant $a$ and $b$ values relating to $\mathrm{Ge}$ detector in Eq. (2).

6) Prediction of $K$ at the furthest position in AOI by EGS5 code.

7) Decision of SPD.

8) In-situ measurement in AOI.

9) Judgment: if Co-60 is not detected within period longer than SPD, it proves that there is no significant Co-60 in AOI.

\section{Conclusion}

The inspection survey method to check radioactivity remaining on the sites of decommissioned NPP was proposed. The in-situ inspection survey test using the portable Ge detector proved that the proposed evaluation method was reasonable for the conservative evaluation.

\section{Acknowledgements}

This work is a part of study funded by Japan Nuclear Energy Safety Organization.

\section{References}

[1] USNRC, Multi-agency Radiation Survey and Site
Investigation Manual (MARSSIM), NUREG-1575, Rev.1, U. S. Nuclear Regulatory Commission (USNRC), (2000).

[2] M. Lindgren, M. Pettersson, M. Wiborgh and A. B. Kemakta, Correlation Factors for C-14,Cl-36, Ni-59, $\mathrm{Ni}-63, \mathrm{Mo}-93, \mathrm{TC}-99, \quad \mathrm{I}-129$ and Cs-135 in Operational Waste for SFR 1, SKB Rapport R-07-05, Swedish Nuclear Fuel and Waste Management Co. (SKB), (2007).

[3] MEXT, In-situ measurement method by using germanium semiconductor detector, Radioactivity Measurement Method Series No. 33, Ministry of Education, Culture, Sports, Science and Technology (MEXT), Tokyo, (2008). [in Japanese]

[4] A. N. Tyler, In situ and airbone gamma-ray spectrometry, in Ed. P. P. Povinec, Analysis of Environmental Radionuclides, Elsevier, Hungary, (2008), pp.407-408.

[5] H. Hirayama, Y. Namio, A. F. Bielajewa, S. J. Wildermana and W. R. Nelson, The EGS5 Code System, KEK Report 2005-8, High Energy Accelerator Research Organization (KEK), (2005).

[6] SSK, Recommendation of the German Commission for Radiation Protection as of 24. 04. 98, German Commission on Radiological Protection (SSK), Bonn, (1998).

[7] The law for the regulations of nuclear source material, nuclear fuel material and reactors, Japan, (1957).

[8] T. Sukegawa, T. Shimada, T. Ito and T. Tanaka, Study on Residual Radioactivity Measurement Method for Site Release of Nuclear Facilities - In-situ Radioactivity Measurement Test with the Use of Portable Ge Semiconductor Detector - (Contract Research), JAEA-Technology 2011-025, Japan Atomic Energy Agency (JAEA), (2011). [in Japanese] 